\title{
Minimum 5-year Follow-up Results of Minimally Invasive Total Knee Arthroplasty Using Mini-Keel Modular Tibial Implant
}

\author{
Ju-Hyung Yoo, $\mathrm{MD}^{1}$, Byoung-Kyu Park, $\mathrm{MD}^{2}$, Chang-Dong Han, $\mathrm{MD}^{2}$, Hyun-Cheol Oh, $\mathrm{MD}^{1}$, and \\ Sang-Hoon Park, $\mathrm{MD}^{1}$ \\ ${ }^{1}$ Department of Orthopedic Surgery, National Health Insurance Service Ilsan Hospital, Goyang; ${ }^{2}$ Department of Orthopedic Surgery, Yonsei University College of \\ Medicine, Seoul, Korea
}

\begin{abstract}
Purpose: To evaluate the minimum 5-year mid-term clinical and radiological results of minimally invasive surgery total knee arthroplasty (MIS-TKA) using a mini-keel modular tibia component.

Materials and Methods: We retrospectively evaluated 254 patients (361 cases) who underwent MIS-TKA between 2005 and 2006. The latest clinical and radiological assessments were done in 168 cases that had been followed on an outpatient basis for more than 5 postoperative years. Clinical results were assessed using the Hospital for Special Surgery (HSS) score and Knee Society score. Radiological evaluation included measurements of knee alignment.

Results: The average postoperative knee range of motion and HSS score were $134.3^{\circ} \pm 12.4^{\circ}$ and $92.7^{\circ} \pm 7.0^{\circ}$, respectively. The average postoperative femorotibial angle and tibial component alignment angle were $5.2^{\circ} \pm 1.7^{\circ}$ valgus and $90.2^{\circ} \pm 1.6^{\circ}$, respectively. The average tibial component posterior inclination was $4.8^{\circ} \pm 2.1^{\circ}$. The percentage of cases with tibial component alignment angle of $90^{\circ} \pm 3^{\circ}$ was $96.1 \%$, and that with the femorotibial angle of $6^{\circ} \pm 3^{\circ}$ valgus was $94.0 \%$. Radiolucent lines were observed in 20 cases (12.0\%): around the femur, tibia, and patella in 14 cases, 10 cases, and 1 case, respectively. However, they were less than $2 \mathrm{~mm}$ and non-progressive in all cases. The survival rate was $99.4 \%$ and there was no implant-related revision.

Conclusions: MIS-TKA using a mini-keel modular tibial plate showed satisfactory results, a high survival rate, and excellent clinical and radiological results in the mid-term follow-up.
\end{abstract}

Keywords: Knee, Arthroplasty, Minimally invasive, Mini-keel tibial component

\section{Introduction}

Minimally invasive surgery total knee arthroplasty (MIS-TKA) requires downsizing or modification of surgical equipment due to the small field of view; medial/lateral or proximal/distal move-

Received January 2, 2014; Revised (1st) April 24, 2014; (2nd) May 9, 2014; (3rd) May 19, 2014; (4th) June 3, 2014; Accepted June 16, 2014

Correspondence to: Sang-Hoon Park, MD

Department of Orthopedic Surgery, National Health Insurance Service Ilsan Hospital, 100 Ilsan-ro, Ilsandong-gu, Goyang 410-719, Korea

Tel: +82-31-900-0540, Fax: +82-31-900-0343

E-mail: orthomania@gmail.com

This is an Open Access article distributed under the terms of the Creative Commons Attribution Non-Commercial License (http://creativecommons.org/licenses/by-nc/3.0/) which permits unrestricted non-commercial use, distribution, and reproduction in any medium, provided the original work is properly cited. ment of a retractor to secure an adequate field of view; extension/ flexion or medial/lateral rotation of the knee joint during surgery; and, above all, continuous development of surgical techniques and cooperation of the members of a surgical team ${ }^{1-5)}$. In addition, the use of an implant designed specifically for MIS can improve the efficiency of the surgery. The mini-keel modular tibial implant (Nexgen MIS Tibial Component; Zimmer Inc., Warsaw, IN, USA) is one of those inventions that can be inserted into the knee in flexed position without anterior dislocation of the knee joint due to the presence of a short keel in the tibial component (Fig. 1) $)^{6}$.

The purpose of this study was to evaluate the efficacy and longevity of the mini-keel modular tibial implant in MIS-TKA based on a retrospective review of clinical and radiological findings. 

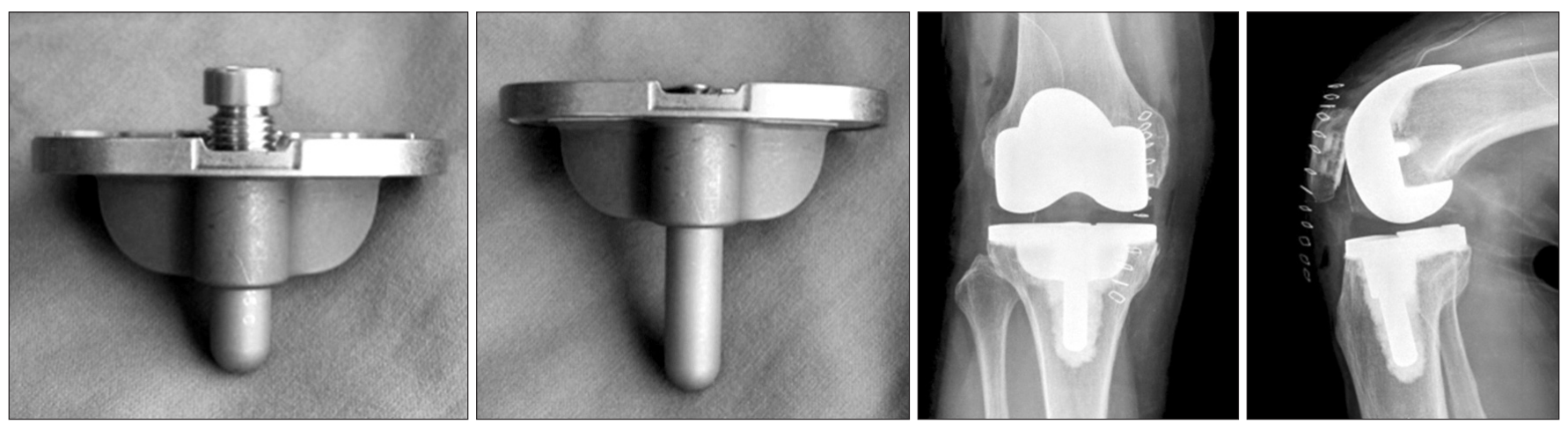

Fig. 1. Modular tibial implants are composed of NexGen MIS Precoat Stemmed Tibial Plates \& drop-down stem extensions.

\section{Materials and Methods}

\section{Patients}

A total of 375 TKAs were performed by the same surgeon at our institution between November 2005 and October 2006. Of those, 361 TKAs were performed via minimally invasive approach in 254 patients, and surgical outcomes and intraoperative complications were investigated among these patients. Clinical and radiological assessments were performed in patients who were available for more than 5-year follow-up. In all cases, the Nexgen Legacy Posterior Stabilized Flex Fixed Bearing (LPS Flex Fixed, Zimmer Inc.) and the Nexgen MIS Tibial Component were used. The mean age of the patients was $68.8+5.6$ years (range, 53 to 80 years). The mean body mass index was $27 \pm 3.7 \mathrm{~kg} / \mathrm{m}^{2}$ (range, 20.6 to $36.8 \mathrm{~kg} / \mathrm{m}^{2}$ ). The indications for surgery were degenerative arthritis in 165 cases, rheumatoid arthritis in 2 cases, and osteonecrosis in 1 case. Preoperatively, the mean range of motion (ROM) was $125.2^{\circ} \pm 17.7^{\circ}$ (range, $70^{\circ}$ to $145^{\circ}$ ), the mean knee score was $65.0 \pm 7.6$ points (range, 22 to 82 points), and the mean femorotibial angle was $4.0^{\circ} \pm 5.6^{\circ}$ varus (range, $21^{\circ}$ varus to $2^{\circ}$ valgus).

Clinical and radiological assessments were conducted at the last follow-up in 168 cases that were available for more than 5-year follow-up on an outpatient basis. One hundred and forty- one cases that were available for a phone interview only in January 2013 were excluded from the clinical evaluation and so were the 79 cases (due to death in 23, nursing home admission in 10, intertrochanteric fracture in 4 , distal femur fracture in 3 , transtibial amputation in 1 , infection-related revision in 1 , immigration to a foreign country in 1 , and loss of contact in 36 cases).

\section{Surgical Technique and Rehabilitation}

A curved skin incision was made from $5 \mathrm{~mm}$ above the medial patella to the superior aspect of the medial tibial tuberosity. In cases where severe skin retraction was observed or there was dif- ficulty with the operation or implant insertion, the skin incision was extended gradually proximally or distally to avoid excessive tension on the skin. Then, the joint was excised using the minimidvastus approach ${ }^{7,8)}$ : a $1.5 \mathrm{~cm}$ proximal incision was started superomedial to the patella in parallel to the vastus medialis muscle fibers and extended along the medial patella to $3 \mathrm{~cm}$ distal to the knee joint surface. Subsequently, patellar osteotomy was performed to obtain sufficient field of view. The patella was resurfaced in all cases. A $6^{\circ}$ valgus intramedullary alignment rod attached to a distal cutting guide was inserted into the center of the knee joint where the anatomical axis of the femur passes though. With the distal cutting guide pin fixed on the medial aspect of the medial femoral condyle, a distal femoral cut was made on the medial aspect of the femur.

A proximal tibial cut was performed using an extramedullary alignment rod as in conventional TKA. Using the cutting guide, the osteotomy was initiated on the anteromedial aspect of the tibia and extended to involve approximately more than $80 \%$ of the proximal tibia except for some posterolateral and lateral areas. Due to the limited field of view, the osteotomy in the posterolateral aspect was performed using a free hand technique.

The rotational alignment of the femur was determined in reference to the posterior condylar axis. At the same time, the appearance of the 'grand-piano sign ${ }^{99}$ on the anterior resected surface of the femur was confirmed before resection.

With the tibial component temporarily press-fitted, a $45 \mathrm{~mm}$ drop-down stem extension was additionally inserted to increase the contact surface between the tibial component and the tibia and fixation strength.

For the 3 postoperative days, a deep vein thrombosis (DVT) foot pump was used to prevent DVT without administration of thrombolytic agents. Immediately postoperatively, knee joint exercises using a passive motion machine were initiated. Within 2 postoperative days, ambulation was permitted depending on the 
patient's condition.

\section{Clinical and Radiological Evaluation}

On the postoperative clinical evaluation, the Hospital for Special Surgery (HSS) score, Knee Society score (KSS) ${ }^{10)}$, and ROM were assessed at the last follow-up in the 168 cases that were available for more than 5-year follow-up. On the radiological evaluation, the femorotibial angle, tibial component alignment angle, and tibial component posterior inclination were assessed on the standing anteroposterior and lateral views taken at the last follow-up according to the Knee Society Total Knee Arthroplasty Roentgenogrphic Evaluation ${ }^{11)}$. In addition, implant location and fixation status were assessed. To assess the implant fixation status, the bone-cement or cement-implant contact area was divided into 7 zones on the lateral view for the femoral component, whereas into 7 zones on the anteroposterior view and 3 zones on the lateral view for the tibial component. The width of radiolucent lines on 5 different zones on the skyline view was measured to assess the stability of the patellar component. Presence of a $\geq 2$-mm-wide radiolucent line, implant subsidence, or change in the alignment was considered as a sign of radiological loosening.

\section{Statistical Analysis}

SPSS ver. 12.0 (SPSS Inc., Chicago, IL, USA) was used for sta- tistical analysis. Preoperative and postoperative clinical findings were compared using a t-test with statistical significance set at $\mathrm{p}<0.05$. Kaplan-Meier analysis was used to estimate the implant survival rate.

\section{Results}

\section{Clinical and Radiological Results}

The mean follow-up period was 6 years and 1 month (minimum, 5 years; maximum, 7 years and 7 months). The mean postoperative femorotibial angle was $5.2^{\circ} \pm 1.7^{\circ}$ valgus (range, $2.6^{\circ}$ to $8.1^{\circ}$ ) and the mean tibial component alignment angle was $90.2^{\circ} \pm 1.6^{\circ}$ varus (range, $86.5^{\circ}$ to $93.4^{\circ}$ ). The mean tibial component posterior inclination was $4.8^{\circ} \pm 2.1^{\circ}$ (range, $1.9^{\circ}$ to $8.1^{\circ}$ ). The femorotibial alignment angle was $6^{\circ} \pm 3^{\circ}$ valgus in 340 of the 361 cases (94.0\%) and the tibial component alignment angle was $0 \pm 3^{\circ}$ varus in 347 cases (96.1\%) (Table 1).

The mean ROM was increased from $125.2^{\circ} \pm 17.7^{\circ}$ (range, $70^{\circ}$ to $145^{\circ}$ ) preoperatively to $134.3^{\circ} \pm 12.4^{\circ}$ (range, $85^{\circ}$ to $145^{\circ}$ ) postoperatively. The mean postoperative KSS was $94.5 \pm 5.9$ points (range, 68 to 100 points), function score was $84.8 \pm 15.6$ points (range, 54 to 100 points), and HSS was $92.7 \pm 7.0$ points (range, 62 to 100 points), all of which were significantly improved after surgery (Table 2).

Table 1. Postoperative Data

\begin{tabular}{lc}
\hline \multicolumn{1}{c}{ Characteristic } & Mean \pm standard deviation (range) \\
\hline Operation time $(\mathrm{min})$ & $87.5 \pm 12.4(57-123)$ \\
Incision length $(\mathrm{cm})$ & $8.3 \pm 0.7(5.8-10)$ \\
Total blood loss $(\mathrm{mL})$ & $1076 \pm 339(250-1810)$ \\
Femorotibial angle $\left(^{\circ}\right)$ & $5.2 \pm 1.7$ valgus $(2.6$ valgus -8.1 valgus $)$ \\
Tibial component alignment angle $\left(^{\circ}\right)$ & $0.2 \pm 1.6$ varus $(3.5$ varus-3.4 valgus $)$ \\
Tibial component posterior inclination $\left(^{\circ}\right)$ & $4.8 \pm 2.1(1.9-8.1)$ \\
Postoperative femorotibial angle in $6^{\circ} \pm 3^{\circ}$ valgus & $94.0 \%$ \\
Tibial component alignment angle in $0^{\circ} \pm 3^{\circ}$ & $96.1 \%$ \\
\hline
\end{tabular}

Table 2. Minimum Postoperative 5-Year Clinical Assessment ( $\mathrm{n}=168)$

\begin{tabular}{lcc}
\hline \multicolumn{1}{c}{ Variable } & Preoperative & Last F/U \\
\hline Range of motion $\left({ }^{\circ}\right)$ & $125.2 \pm 17.7$ & $134.3 \pm 12.4$ \\
Knee Society knee score & $38.4 \pm 13.9$ & $94.5 \pm 5.9$ \\
Knee Society functional score & $47.9 \pm 11.6$ & $84.8 \pm 15.6$ \\
Hospital for Special Surgery score & $64.9 \pm 7.4$ & $92.7 \pm 7.0$ \\
\hline
\end{tabular}

Values are presented as mean \pm standard deviation. p-value $<0.001$.

F/U: follow-up.
Table 3. Incidence of Radiolucent Lines in Each Radiographic Zone

\begin{tabular}{lccccccc}
\hline \multirow{2}{*}{\multicolumn{1}{c}{ Site }} & \multicolumn{7}{c}{ Zone (case) } \\
\cline { 2 - 8 } & 1 & 2 & 3 & 4 & 5 & 6 & 7 \\
\hline Femur & 8 & 1 & 0 & 8 & 0 & 0 & 0 \\
Tibia & & & & & & & \\
$\quad$ Anteroposterior & 9 & 3 & 0 & 1 & 1 & 0 & 1 \\
$\quad$ Lateral & 0 & 0 & 2 & & & & \\
Patella & 1 & 0 & 0 & & & & \\
\hline
\end{tabular}




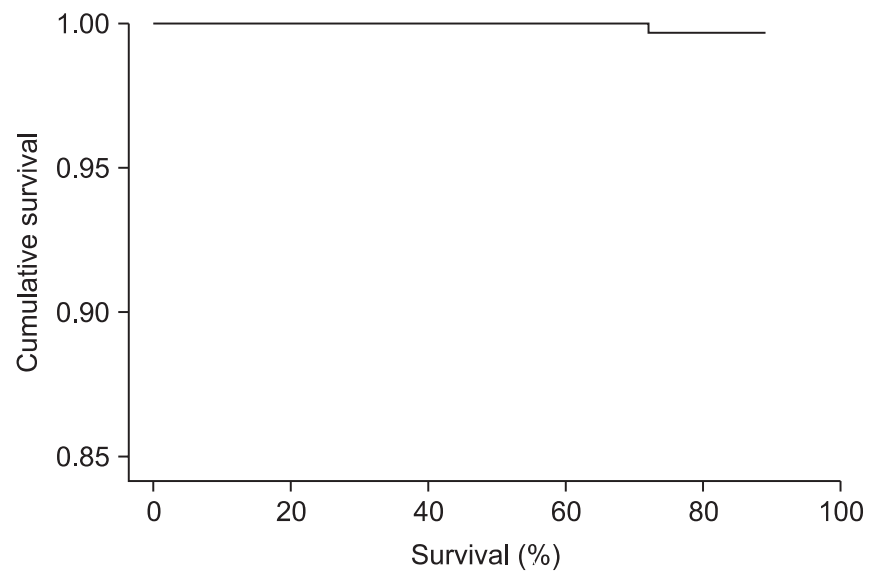

Fig. 2. Kaplan-Meier survival analysis.

Revision was not necessary in all cases based on the last followup radiological assessment which showed no evidence of femoral/tibial component alignment change, subsidence, or loosening. Radiolucency was observed in more than one zone in the boneimplant interface in 22 cases (13.1\%) at the last follow-up: in the femur and tibia both in 4 cases, in the femur only in 10 cases, in the tibia only in 7 cases, and in the patella only in 1 case. However, all of which were less than 2-mm non-progressive radiolucent lines. The components demonstrated stable fixation without signs of alignment changes or loosening in all cases. The radiolucency was observed in 1 zone in 15 cases, in 2 zones in 4 cases, and in more than 3 zones in 3 cases. In the femur, it was located in zone 1 in 8 cases, in zone 2 in 1 case, and in zone 4 in 8 cases. In the tibia, it was observed in zone 1 in 9 cases, in zone 2 in 3 cases, and in zones 4,5, and 7 in 1 case each on the anteroposterior view whereas in zone 3 in 2 cases on the lateral view. In the patella, it was found in zone 1 in 1 case (Table 3).

\section{Survivorship}

The mean survival rate per Kaplan-Meier analysis was $99.4 \%$ at 6.1 years (Fig. 2). Revision was required due to component loosening in one of the 168 cases that were followed for more than 5 years.

\section{Discussion}

Determining whether MIS is an appropriate treatment option in TKA requires assessments on its conformity to standard operating principles, such as flexion/extension gap balancing, restoration of proper alignment, prevention of intraoperative complications, and achievement of expected surgical outcome $e^{1,2,712-15)}$.

In the current study, the MIS-TKA demonstrated relatively high accuracy: the tibial component alignment angle was $0^{\circ} \pm 3^{\circ}$ varus in 347 of the 361 cases (96.1\%) and the femorotibial angle was $6^{\circ} \pm 3^{\circ}$ valgus in 340 cases $(94.0 \%)$. This can be attributed to the proper placement of the cutting alignment guide during surgery in spite of the limited field of view, considering that femoral and tibial alignments are dictated by the intramedullary/extramedullary alignment guide. During surgery, we expected the side cutting technique for distal femoral cut would result in minimal soft tissue damage and verified firm attachment of the distal cutting guide to the $6^{\circ}$ valgus intramedullary alignment rod for precise osteotomy in the medial condyle of the distal femur. For lateral femoral resection, this intramedullary alignment rod should be removed: thus, the remaining distal cutting guide on the medial aspect of the medial femoral condyle can be displaced by soft tissue during the procedure. In addition, the osteotomy should be carried out in the absence of proper field of view, rendering it difficult to conduct precise resection. To overcome this obstacle, we removed the distal cutting guide as well and used a free hand technique for lateral femoral condyle resection by referring to the resected surface of the medial femoral condyle.

With experience, MIS-TKA can be performed through a small incision without applying excessive tension on the surrounding soft tissue: however, implant insertion can still be challenging if the field of view is extremely limited ${ }^{1,3,4,14)}$. In order to insert conventional tibial components that have long stems, the tibial resection surface should be sufficiently anteriorly dislocated relative to the femoral resection surface with the knee in flexion. This may be not feasible if the incision is small, result in complications caused by excessive tension on the adjacent tissue, or require additional soft tissue resection. The modular tibial implant (NexGen MIS Precoat Stemmed Tibial Plate \& drop-down stem extensions) was designed to overcome these disadvantages by allowing implant insertion in knee flexion without anterior dislocation of the tibia through a small field of view.

The modular tibial implant is made of titanium alloy and composed of a mini-keel tibial plate and a $45 \mathrm{~mm}$ - or $75 \mathrm{~mm}$ long modular stem (drop-down stem extension). The short keel length (range, 17 to $19 \mathrm{~mm}$ ) in the tibial plate facilitates insertion into a flexed knee without anterior dislocation of the tibia against the femur, thus preventing tension on the surrounding tissue and additional soft tissue resection. However, care should be taken during insertion because the keel should be passed through a narrow small space in the flexed knee, and determining the exact insertion site can be challenging because the keel punch in the resected surface of the tibia is not easy to identify due to cement. Therefore, we applied sufficient traction with the knee in flexed 
position during mini-keel plate insertion and used a custom made holder to securely hold and precisely insert the implant through a narrow space. Although the length of the keel of the mini-keel tibial component is short compared to that of other tibial components, it provides $5.20 \%$ wider contact surface, thus not compromising stability of the knee. However, in an attempt to obtain further stability by increasing the interface between the implant and the tibia, we additionally used a $45 \mathrm{~mm}$ drop-down stem extension in all knees.

Benazzo and Rossi ${ }^{(6)}$ first reported on the results of MIS-TKA using a modular tibial implant: the 5-year survival rate was $97.9 \%$ in 200 knees during the 3-year follow-up in the prospective study. Bonutti et al. ${ }^{2)}$ demonstrated favorable clinical and radiological results: the 9-year survival rate was $97.1 \%$ in 90 knees. In the current study, the survival rate was as high as $99.4 \%$ at 6.1 years after MIS-TKA using a mini-keel tibial implant, which can be attributed to appropriate realignment of the tibial component, proper selection for tibial fixation site, and the use of a drop-down stem extension for improved stability.

Seo et al. ${ }^{5)}$ documented that surgical site healing was delayed and the incidence of infection was high after MIS-TKA that was performed under limited visualization of the operative field compared to that after conventional TKA due to severe skin traction and prolonged surgical time. However, there was no case of infection except for 1 case that required revision due to infection at 6 years and 6 months after surgery. The infection rate in our patients was significantly low compared to that in previous studies, which we believe is attributable to careful application of traction to skin and the relatively short surgical time $(87.5 \pm 12.4 \mathrm{~min})$.

Most publications in the literature are reports on the short-term results of MIS-TKA using the conventional implants ${ }^{1,4,9,13,15-17)}$. Thus, the significance of this study can be found in the fact that we investigated more than 5-year mid-term follow-up results of MIS-TKA using a tibial component designed specifically for MIS in a relatively large study population.

The limitations of this study include that the study design was retrospective and many of the 316 cases that underwent MISTKA were not included for analysis due to unavailability for outpatient clinic evaluation. In addition, all the operations were performed by the same surgeon experienced in MIS-TKA. Although the procedure requires a long learning curve to achieve accuracy and reduce operation time ${ }^{1,8)}$, we think that long-term studies that involve multiple institutions and various surgeons should be conducted in the near future. Furthermore, comparison with the conventional TKA implants should be addressed in future studies.

\section{Conclusions}

The more than 5-year mid-term follow-up of MIS-TKA using a mini-keel modular tibial implant in 168 cases showed high implant survivorship and satisfactory clinical and radiological results.

\section{Conflict of Interest}

No potential conflict of interest relevant to this article was reported.

\section{References}

1. Barrack RL, Barnes CL, Burnett RS, Miller D, Clohisy JC, Maloney WJ. Minimal incision surgery as a risk factor for early failure of total knee arthroplasty. J Arthroplasty. 2009; 24:489-98.

2. Bonutti PM, Costa CR, Woehnl A, Johnson AJ, Mont MA. Results of MIS TKA at mean nine year follow-up. J Knee Surg. 2011;24:203-7.

3. Dalury DF, Dennis DA. Mini-incision total knee arthroplasty can increase risk of component malalignment. Clin Orthop Relat Res. 2005;440:77-81.

4. King J, Stamper DL, Schaad DC, Leopold SS. Minimally invasive total knee arthroplasty compared with traditional total knee arthroplasty. Assessment of the learning curve and the postoperative recuperative period. J Bone Joint Surg Am. 2007;89:1497-503.

5. Seo JG, Moon YW, Jo BC, Kim YT, Park SH. soft tissue balancing of varus arthritic knee in minimally invasive surgery total knee arthroplasty: comparison between posterior oblique ligament release and superficial MCL release. Knee Surg Relat Res. 2013;25:60-4.

6. Benazzo F, Rossi SM. Modular tibial plate for minimally invasive total knee arthroplasty. Knee Surg Sports Traumatol Arthrosc. 2012;20:1796-802.

7. Gandhi R, Smith H, Lefaivre KA, Davey JR, Mahomed NN. Complications after minimally invasive total knee arthroplasty as compared with traditional incision techniques: a meta-analysis. J Arthroplasty. 2011;26:29-35.

8. Nestor BJ, Toulson CE, Backus SI, Lyman SL, Foote KL, Windsor RE. Mini-midvastus vs standard medial parapatellar approach: a prospective, randomized, double-blinded study in patients undergoing bilateral total knee arthroplasty. J Arthroplasty. 2010;25(6 Suppl):5-11. 
9. Cui WQ, Won YY, Baek MH, Kim KK, Cho JH. Variations of the 'grand-piano sign' during total knee replacement: a computer-simulation study. J Bone Joint Surg Br. 2006;88:1441-7.

10. Ewald FC. The Knee Society total knee arthroplasty roentgenographic evaluation and scoring system. Clin Orthop Relat Res. 1989;(248):9-12.

11. Insall JN, Dorr LD, Scott RD, Scott WN. Rationale of the Knee Society clinical rating system. Clin Orthop Relat Res. 1989;(248):13-4.

12. Cheng T, Liu T, Zhang G, Peng X, Zhang X. Does minimally invasive surgery improve short-term recovery in total knee arthroplasty? Clin Orthop Relat Res. 2010;468:1635-48.

13. Lin SY, Chen CH, Fu YC, Huang PJ, Lu CC, Su JY, Chang JK, Huang HT. Comparison of the clinical and radiological outcomes of three minimally invasive techniques for total knee replacement at two years. Bone Joint J. 2013;95:906-10.
14. Niki Y, Matsumoto H, Otani T, Enomoto H, Toyama Y, Suda Y. Accuracy of implant positioning for minimally invasive total knee arthroplasty in patients with severe varus deformity. J Arthroplasty. 2010;25:381-6.

15. Tanavalee A, Thiengwittayaporn S, Itiravivong P. Progressive quadriceps incision during minimally invasive surgery for total knee arthroplasty: the effect on early postoperative ambulation. J Arthroplasty. 2007;22:1013-8.

16. Labek G, Thaler M, Janda W, Agreiter M, Stockl B. Revision rates after total joint replacement: cumulative results from worldwide joint register datasets. J Bone Joint Surg Br. 2011; 93:293-7.

17. Watanabe T, Muneta T, Ishizuki M. Is a minimally invasive approach superior to a conventional approach for total knee arthroplasty? Early outcome and 2- to 4-year follow-up. J Orthop Sci. 2009;14:589-95. 